\title{
Vorwort
}

\section{zur 1. Auflage}

Die Fachbuchreihe Baukonstruktionen mit ihren 17 Basisbänden stellt eine Zusammenfassung des derzeitigen technischen Wissens bei der Errichtung von Bauwerken des Hochbaues dar. Es wird versucht, mit einfachen Zusammenhängen oft komplexe Bereiche des Bauwesens zu erläutern und mit zahlreichen Plänen, Skizzen und Bildern zu veranschaulichen.

Dieser Band behandelt den Bauteil "Fenster" als Öffnung in der Wand. Ausgehend von einer architektonischen Betrachtung wird ein struktureller Überblick der gebräuchlichen Fenstertypen gegeben. Das Fenster wird systematisch nach seinen Bauelementen, konstruktiv, normativ und bauphysikalisch betrachtet. Dabei wird versucht, der technologischen Entwicklung Rechnung tragend, die Vielzahl an modernen Fensterkonstruktionen und deren Baukörperanschlüssen in eine Genealogie des Fensters einzureihen.

Der Herausgeber

\section{zur 2. Auflage}

Die Fachbuchreihe Baukonstruktionen mit ihren 17 Basisbänden stellt eine Zusammenfassung des derzeitigen technischen Wissens bei der Errichtung von Bauwerken des Hochbaus dar. Durch die laufenden Änderungen an der Normung und den gesetzlichen Vorgaben sowie die technische Weiterentwicklung sind Anpassungen der Inhalte erforderlich geworden. In dieser Auflage wurde versucht, weitgehend die aktuellen Inhalte der Normen und behördlichen Vorschreibungen aufzunehmen, angefangen von den Nachweisführungen der Verglasungen bis zu notwendigen Leistungsmerkmalen für die Vermarktung von Bauprodukten, die durch Leistungserklärungen als wesentliche Merkmale der Bauprodukte - eine CE-Kennzeichnung - auch für Fenster und Fenstertüren geregelt sind. Im Speziellen sind auch neue Anforderungen an den Einbau von Fensterelementen behandelt, da hier in der Praxis die meisten Probleme und erhebliche Schadenspotentiale vorliegen. Bedingt durch Covid19 und die damit verbundenen Betriebseinschränkungen, war es bis zum Redaktionsschluss leider nicht möglich für alle Bilddarstellungen Aktualisierungen zu erhalten.

Der Herausgeber 